\title{
DFAST and DAGA: web-based integrated genome annotation tools and resources
}

\author{
Yasuhiro TANIZAWA ${ }^{1,2}$, Takatomo FUJISAWA ${ }^{2}$, Eli KAMINUMA ${ }^{2}$, Yasukazu NAKAMURA ${ }^{2}$ and \\ Masanori ARITA ${ }^{2,3^{*}}$ \\ ${ }^{1}$ Department of Computational Biology and Medical Sciences, Graduate School of Frontier Sciences, The University of Tokyo, \\ 5-1-5 Kashiwanoha, Kashiwa, Chiba 277-8561, Japan \\ ${ }^{2}$ Center for Information Biology, National Institute of Genetics, 1111 Yata, Mishima, Shizuoka 411-8540, Japan \\ ${ }^{3}$ RIKEN Center for Sustainable Resource Science, 1-7-22 Suehiro-cho, Tsurumi, Yokohama, Kanagawa 230-0045, Japan
}

Received February 15, 2016; Accepted June 27, 2016; Published online in J-STAGE July 14, 2016

Quality assurance and correct taxonomic affiliation of data submitted to public sequence databases have been an everlasting problem. The DDBJ Fast Annotation and Submission Tool (DFAST) is a newly developed genome annotation pipeline with quality and taxonomy assessment tools. To enable annotation of ready-to-submit quality, we also constructed curated reference protein databases tailored for lactic acid bacteria. DFAST was developed so that all the procedures required for DDBJ submission could be done seamlessly online. The online workspace would be especially useful for users not familiar with bioinformatics skills. In addition, we have developed a genome repository, DFAST Archive of Genome Annotation (DAGA), which currently includes 1,421 genomes covering 179 species and 18 subspecies of two genera, Lactobacillus and Pediococcus, obtained from both DDBJ/ENA/GenBank and Sequence Read Archive (SRA). All the genomes deposited in DAGA were annotated consistently and assessed using DFAST. To assess the taxonomic position based on genomic sequence information, we used the average nucleotide identity (ANI), which showed high discriminative power to determine whether two given genomes belong to the same species. We corrected mislabeled or misidentified genomes in the public database and deposited the curated information in DAGA. The repository will improve the accessibility and reusability of genome resources for lactic acid bacteria. By exploiting the data deposited in DAGA, we found intraspecific subgroups in Lactobacillus gasseri and Lactobacillus jensenii, whose variation between subgroups is larger than the well-accepted ANI threshold of $95 \%$ to differentiate species. DFAST and DAGA are freely accessible at https://dfast.nig.ac.jp.

Key words: lactic acid bacteria, genome, annotation, database, Lactobacillus, Pediococcus

\section{INTRODUCTION}

Major scientific journals request that researchers deposit newly sequenced DNA in the International Nucleotide Sequence Database Collaboration (INSDC) [1]. DDBJ/ENA/GenBank are the core annotation databases, collecting publicly available DNA information with metadata. Recently, INSDC has also begun collecting raw sequences from the new-generation sequencing platforms for Sequence Read Archive (SRA) [2]. These primary public databases constitute the basis

\footnotetext{
*Corresponding author. Masanori Arita, Center for Information Biology, National Institute of Genetics, 1111 Yata, Mishima, Shizuoka 411-8540, Japan. Phone: +81-55-981-9449; E-mail: arita@ nig.ac.jp

(C)2016 BMFH Press

This is an open-access article distributed under the terms of the Creative Commons Attribution Non-Commercial No Derivatives (bync-nd) License $<$ http://creativecommons.org/licenses/by-nc-nd/4.0/>.
}

for accessibility, reproducibility, and reusability of genomic data. However, since quality assurance and correct assignment of taxonomy are the responsibility of data contributors, improving quality and taxonomic description has been an everlasting problem [3-5]. Lowquality data not only decrease the reliability of future analyses but also, in the worst case, lead to biologically incorrect conclusions. To avoid such problems, several tools and methods are available. QUAST [6] is a widely used assessment tool for genome assembly that reports statistical metrics such as N50 and detects misassemblies by using a reference genome. CheckM [7] estimates genome completeness and contamination by inspecting for the presence/absence of marker genes specific to each taxon. To confirm taxonomic affiliation of unidentified genomes, Bull et al. proposed using 16S rRNA genes together with housekeeping genes [4]. Beaz-Hidalgo et al. recommended the use of average nucleotide identity (ANI) to verify the taxonomic position of newly obtained 
genomes [8]. ANI represents the mean sequence identity of homologous regions between a given pair of genomes, and an ANI value of $95-96 \%$ is widely accepted as the threshold for distinguishing species [9-11]. Examples of ANI values and the 16S rRNA gene sequences for curated genomes can be available at the EzGenome and EzTaxon databases [12]. Recently, the use of genomic comparison methods including ANI was also proposed to find and correct misidentified genomes in the public databases at an NCBI workshop [13].

Along this line of research, we developed the DDBJ Fast Annotation and Submission Tool (DFAST) as a web-based bacterial annotation pipeline with integrated quality assessment using CheckM and taxonomic assessment using ANI. DFAST allows researchers to submit annotated genomes easily to INSDC through the DDBJ Mass Submission System (MSS) [14]. As the initial showcase of DFAST, we targeted lactic acid bacteria (LAB) and constructed a reference protein database tailored for Lactobacillus as well as Pediococcus to enable accurate and rapid annotation. We also developed an associated repository, DFAST Archive of Genome Annotation (DAGA), which stores LAB genomes obtained from DDBJ/ENA/GenBank and SRA with consistent annotation and assessment by DFAST. Our aim is to provide a reliable genome resource to the entire research community, thereby promoting accessibility and reusability of genomic data.

Among LAB, Lactobacillus is highly heterogeneous and the largest genus in the family Lactobacillaceae, comprising 185 species and 18 subspecies as of June 2016 (http://www.bacterio.net/lactobacillaceae. $\mathrm{html}$ ). The genus Pediococcus is another member of Lactobacillaceae consisting of 11 species, and it is phylogenetically placed within the Lactobacillus cluster, near $L$. plantarum and $L$. brevis $[15,16]$. In a recent study, the term Lactobacillus sensu lato was also proposed to refer to these genera [17]. In both genera, the number of new species described and genomes published have been growing with the improvement of isolation, cultivation, and identification methods as well as sequencing technology (Fig. 1). Nowadays, most type strains have been sequenced and become publicly available through large-scale sequencing projects, such as "Genome sequencing of JCM strains under the NBRP program" in Japan (BioProject ID: PRJDB547), "Lactobacillus in severe early childhood caries" by Sanger Institute, UK (PRJEB3060), and "Genomic characterization of the genus Lactobacillus" in China (PRJNA222257). The results of such projects have enabled genus-wide analyses covering almost $90 \%$ of

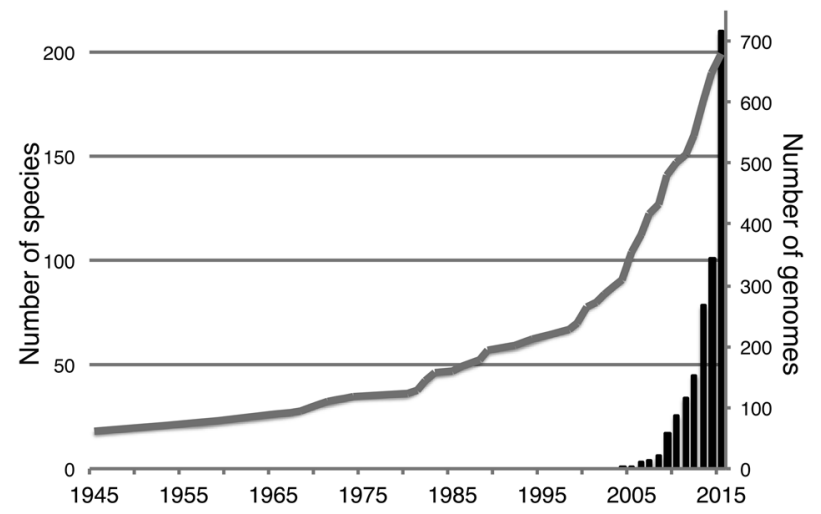

Fig. 1. The number of described species and published genomes in Lactobacillus and Pediococcus.

Solid line represents the cumulative number of described (sub) species. Only valid species as of Jan. 2016 were included, not reclassified ones. The bar chart represents the cumulative number of genomes deposited in DDBJ/ENA/GenBank.

the known species based on the genomic information $[17,18]$. Our DAGA provides annotated genomes for the family Lactobacillaceae, which includes many species that have undergone reclassification and species difficult to distinguish by $16 \mathrm{~S}$ rRNA gene sequences. Our data will benefit all researchers who use LAB genomes, especially those focusing on inter- and intraspecific relations.

In the present article, we describe development of DAGA and DFAST, and we also report several findings related to the current nomenclature.

\section{MATERIALS AND METHODS}

\section{Construction of the annotation pipeline}

The reference protein database was first constructed to provide consistent annotation to all focused genomes. A total of 69 complete genomes of Lactobacillus and Pediococcus, publicly available as of September 2015, were collected from the NCBI Assembly Database, and their protein sequences were extracted. In addition, 12 other genomes were added to link with the Lactobacillales-specific Clusters of Orthologous Genes (LaCOGs) [19] and Microbial Genome Database (MBGD) [20]: Aerococcus urinae ACS120-V-Col10a (GCA_000193205.1), Carnobacterium sp. 17-4 (GCA_000195575.1), Enterococcus faecalis V583 (GCA_000007785.1), Lactococcus lactis subsp. cremoris SK11 (GCA_000014545.1), Lactococcus lactis subsp. lactis I11403 (GCA_000006865.1), Leuconostoc mesenteroides subsp. mesenteroides ATCC 8293 (GCA 000014445.1), Melissococcus plutonius ATCC 
A

DFAST Analysis - Archive

Download About Help

group 9

--- all ---

genus

Lactobacillus $\mathrm{x}$

Update View Download . rating 9

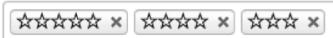

species

paraplantarum $x$ pentosus $x$ plantarum $x$ $\square$ Show only representative genomes. 9

subspecies

-.- disabled

Show Optional Columns: Original Name BioProject BioSample Assembly Level Completeness Contamination

Show $10 \div$ entries

\begin{tabular}{|c|c|c|c|c|}
\hline $\begin{array}{l}\text { ID } \\
\text { (ellek for detail) }\end{array}$ & Organism Name (curated) & $\begin{array}{l}\text { Type } \quad \downarrow \uparrow \\
\text { Status }\end{array}$ & GC\% & $\begin{array}{l}\text { Total } \\
\text { length (bp) }\end{array}$ \\
\hline ERR298627 & Lactobacillus plantarum G226_4_1 & & $44.2 \%$ & $3,480,295$ \\
\hline ERR386058 & Lactobacillus plantarum unkown & & $44.5 \%$ & $3,205,896$ \\
\hline ERR386059 & Lactobacillus plantarum unkown & & $44.5 \%$ & $3,220,634$ \\
\hline ERR387522 & $\begin{array}{l}\text { Lactobacillus plantarum subsp. } \\
\text { argentoratensis DSM } 16365\end{array}$ & type strain & $45.0 \%$ & $3,172,036$ \\
\hline ERR433486 & Lactobacillus paraplantarum LMG_16673 & & $43.7 \%$ & $3,297,581$ \\
\hline ERR433488 & Lactobacillus plantarum DSM 13273 & & $44.3 \%$ & $3,416,139$ \\
\hline ERR485030 & Lactobacillus plantarum G226_5_1 & & $44.2 \%$ & $3,487,612$ \\
\hline ERR485098 & Lactobacillus plantarum G211_1_2 & & $44.3 \%$ & $3,451,941$ \\
\hline ERR485109 & Lactobacillus plantarum G226_2_10 & & $44.3 \%$ & $3,440,375$ \\
\hline ERR570145 & Lactobacillus plantarum G238_1_1 & & $44.4 \%$ & $3,270,238$ \\
\hline
\end{tabular}

Showing 1 to 10 of 85 entries

\begin{tabular}{|c|c|c|c|c|c|c|c|c|}
\hline \multirow[b]{2}{*}{$\begin{array}{l}\text { No. of } \\
\text { Seqs. }\end{array}$} & \multicolumn{8}{|c|}{ Search: } \\
\hline & cDSs & Rating & If & \multicolumn{5}{|c|}{ Note } \\
\hline 142 & 3,271 & \multicolumn{7}{|l|}{ 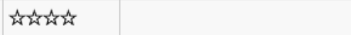 } \\
\hline 35 & 3,000 & \multicolumn{7}{|l|}{ 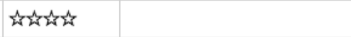 } \\
\hline 29 & 3,019 & \multicolumn{7}{|l|}{ 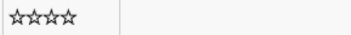 } \\
\hline 148 & 2,939 & \multicolumn{7}{|l|}{ 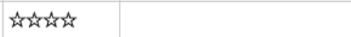 } \\
\hline 249 & 3,069 & \multicolumn{7}{|l|}{ 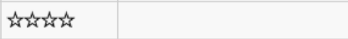 } \\
\hline 77 & 3,242 & \multicolumn{7}{|l|}{ 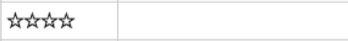 } \\
\hline 140 & 3,281 & \multicolumn{7}{|l|}{ 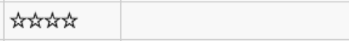 } \\
\hline 97 & 3,245 & \multicolumn{7}{|l|}{ 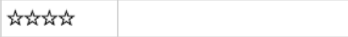 } \\
\hline 140 & 3,238 & \multicolumn{7}{|l|}{ 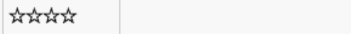 } \\
\hline \multirow[t]{2}{*}{68} & 3,080 & \multicolumn{7}{|l|}{ 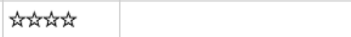 } \\
\hline & Previous & \begin{tabular}{|l|l|}
1 & 2 \\
\end{tabular} & 3 & 4 & 5 & $\ldots$ & 9 & Next \\
\hline
\end{tabular}

B

C

\begin{tabular}{|c|c|c|c|}
\hline \multicolumn{2}{|c|}{ 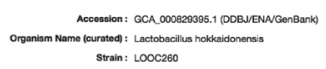 } & \multicolumn{2}{|c|}{ 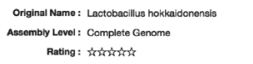 } \\
\hline \multicolumn{4}{|c|}{ 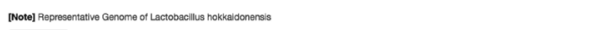 } \\
\hline \begin{tabular}{l|l} 
Sumnay & Features
\end{tabular} & & & \\
\hline \multicolumn{2}{|l|}{ 目 Genome Statistics } & \multirow{13}{*}{ 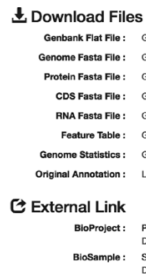 } & \\
\hline Total Longeth (por) & $2,400,566$ & & SCA 000029395.1.90k \\
\hline No. of Saquences & 3 & & GCA_.000829395.1.9enome.t.te \\
\hline ec Content(s) & $38.2 \%$ & & 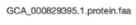 \\
\hline Nso & $2,277,995$ & & GCA $0008203055.1 .0 d$ d.fina \\
\hline Cap Retiof(x) & $0.0 \% 6$ & & GCA_000828396.1.ma.ma \\
\hline No. of Protetens & 2,328 & & 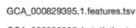 \\
\hline No. of raNA & 12 & & GCA_.000829395. 1.5t \\
\hline No. of trNA & 56 & & \\
\hline No. ot CRISPAS & 1 & & \\
\hline Coding Ratio(\%) & $86.9 \%$ & & PANDB1726 \\
\hline Genome Coverage & $300.0 x$ & & DOBJ/NCBI/EBI \\
\hline Completeness & $99.3 \%$ & & 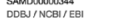 \\
\hline
\end{tabular}

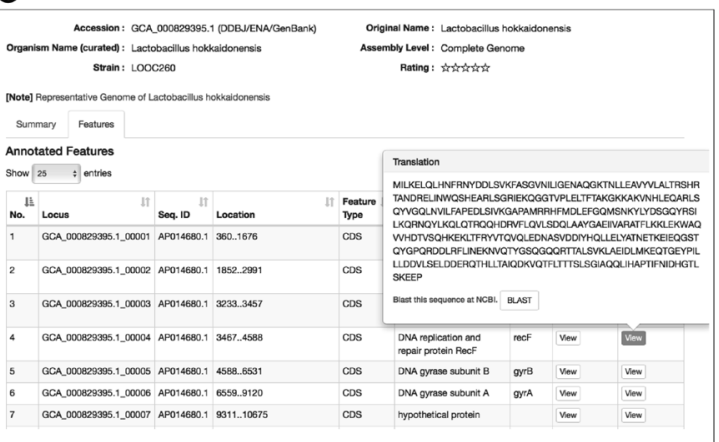

D

$\mathrm{E}$
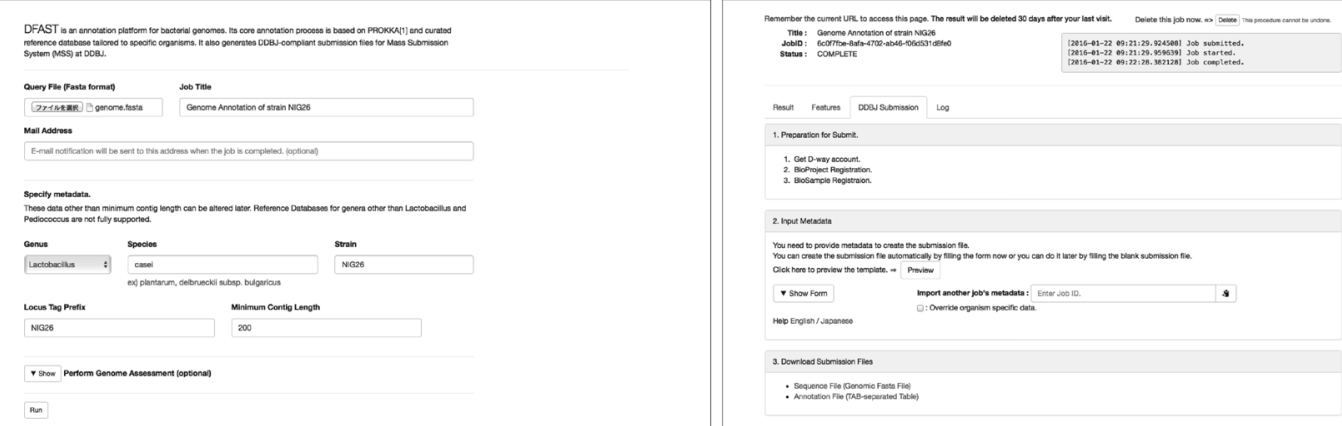

Fig. 2. Screenshots of DFAST and DAGA.

A) Main page of DAGA, listing genomes in the database. Users can query genomes from the search form. B) Detail page of each genome, showing statistics and external links. Data files are downloadable in several formats. C) Detail page of annotated features. Links to the Blast web service at NCBI are available. D) Submission form of DFAST. Users can annotate their own genome by uploading the FASTA file. E) Result of DFAST. Submission files for DDBJ Mass Submission System are ready. 
35311 (GCA_000270185.1), Oenococcus oeni PSU-1 (GCA_000014385.1), Streptococcus pyogenes M1 GAS (GCA_000006785.1), Streptococcus thermophilus LMD-9 (GCA_000014485.1), Tetragenococcus halophilus NBRC 12172 (GCA_000283615.1), and Weissella koreensis KACC 15510 (GCA_000219805.1). The identified 183,469 protein sequences were grouped into 28,002 orthologous clusters by using the GET_HOMOLOGUES software (version 1.3) with its default settings [21]. Briefly, candidates for orthologous genes were determined by bidirectional BLASTP alignments between each pair of the strains with an E-value threshold of 10e-5 and a minimum coverage threshold of $75 \%$. Then, orthologous clusters were detected by the OrthoMCL algorithm [22]. Among them, 11,993 were shared clusters containing two or more protein sequences, and the remaining 16,009 singletons were discarded. To infer the protein names and gene symbols, the shared clusters were mapped to the orthologous clusters of LaCOGs and MBGD. A total of 6,428 clusters were assigned to LaCOGs, of which $98.9 \%$ formed a one-to-one relationship with specific LaCOG clusters. Likewise, an additional 1,601 clusters were assigned to MBGD, of which $94.4 \%$ were oneto-one. To confirm the protein functions, public protein databases and the NCBI Conserved Domain Database [23] were searched manually. All protein names followed the NCBI guidelines for naming proteins (http://www. ncbi.nlm.nih.gov/genbank/genomesubmit_annotation/).

The core annotation process was based on the Prokka annotation software [24], performing prediction of tRNAs, rRNAs, CRISPRs, and protein-coding sequences as well as similarity searches against protein sequence databases and protein family profiles. The reference database was used in our customized Prokka pipeline that can generate DDBJ-compliant submission files.

\section{Data collection}

Publicly available genome sequences for Lactobacillus and Pediococcus were downloaded from the NCBI Assembly Database, which is a secondary database of DDBJ/ENA/Genbank that provides assembled sequences for each genome [25]. Raw sequence data (Illumina sequences with the paired-end method) were downloaded from SRA, and de novo assembly was conducted to reconstruct draft genome sequences as described below. All genomes were annotated with the customized Prokka pipeline.

\section{Genome assembly}

Raw sequence reads were preprocessed using Platanus_trim (version 1.0.7) to remove low-quality regions. De novo assembly was conducted using the Platanus assembler (version 1.2.4) [26]. Since Platanus was originally developed for heterozygous diploid genomes, we specified the parameters "-d 0.3 -u 0.05 " to configure it for bacterial haploid genomes. For each genome, de novo assembly was repeated five times by randomly sampling read sequences of different coverage, and the best result was chosen by the completeness calculated using CheckM and the average sequence length.

\section{Calculation of average nucleotide identity}

The pyani script (https://github.com/widdowquinn/ pyani) was used to calculate the ANI between two genomes, based on the method by Goris et al. [9]. In brief, one genome was cut into 1,020 nt fragments, which were searched against the other genome by using the BLASTN algorithm [27]. ANI was calculated as the mean identity of top-hit BLASTN matches for all fragments with a sequence identity of $\geq 30 \%$ and an overall aligned region of $\geq 70 \%$ of the fragment length. The trees in Fig. 3 (B-D) were constructed by the UPGMA (Unweighted Pair Group Method with Arithmetic Mean) clustering method with a distance of $(1-\mathrm{ANI})$.

\section{Quality assessment of genomes}

CheckM (version 1.0.5) was used to calculate completeness and contamination of each genome [7]. CheckM inspected for the presence/absence of 409 and 664 single-copy gene markers specific for Lactobacillus and Pediococcus, respectively. Genome completeness and contamination were estimated by the number of distinct markers and their multiplicity in each genome, respectively.

\section{Implementation of the web service}

DFAST and DAGA were implemented in Python 2.7.11 with PostgreSQL 8.4.20 and Nginx 1.8.0, and run on a Red Hat Enterprise Linux server (release 6.7).

\section{RESULTS}

\section{Overview of the DAGA service}

We developed an integrated genome archive specialized for LAB, namely DAGA. The first version of the dataset targets the family Lactobacillaceae and contains 1,389 and 32 genomes for Lactobacillus and Pediococcus, respectively. Among them, 743 are publicly available genome sequences deposited in DDBJ/ENA/ GenBank; they were obtained from the NCBI Assembly Database. The remaining 678 genomes were assembled 
A

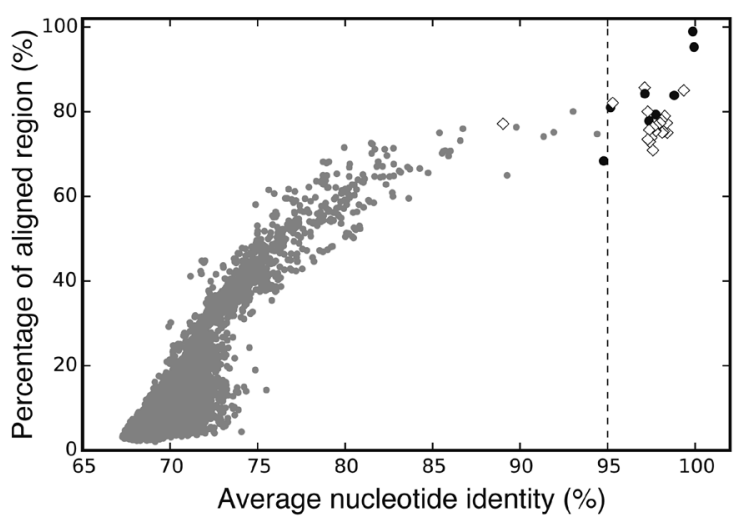

C

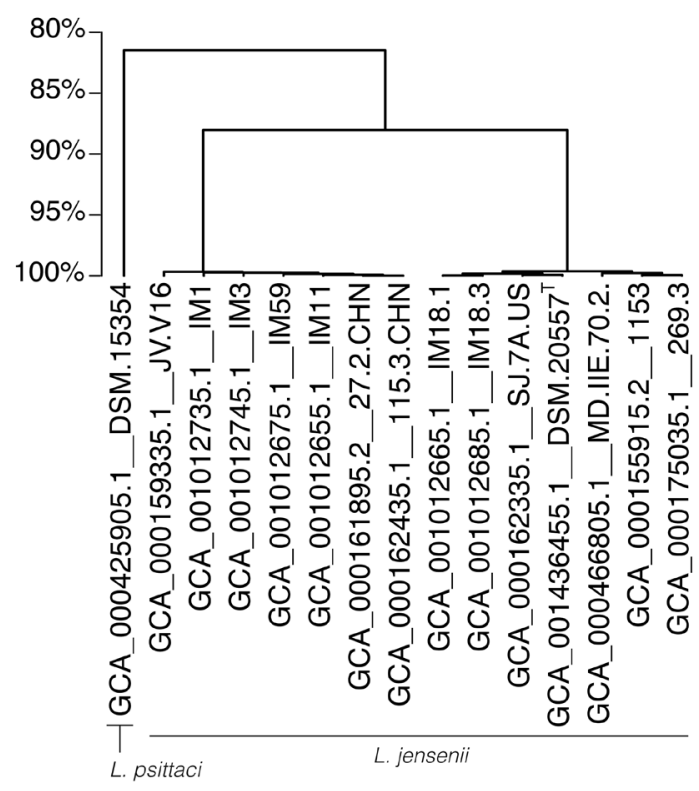

B
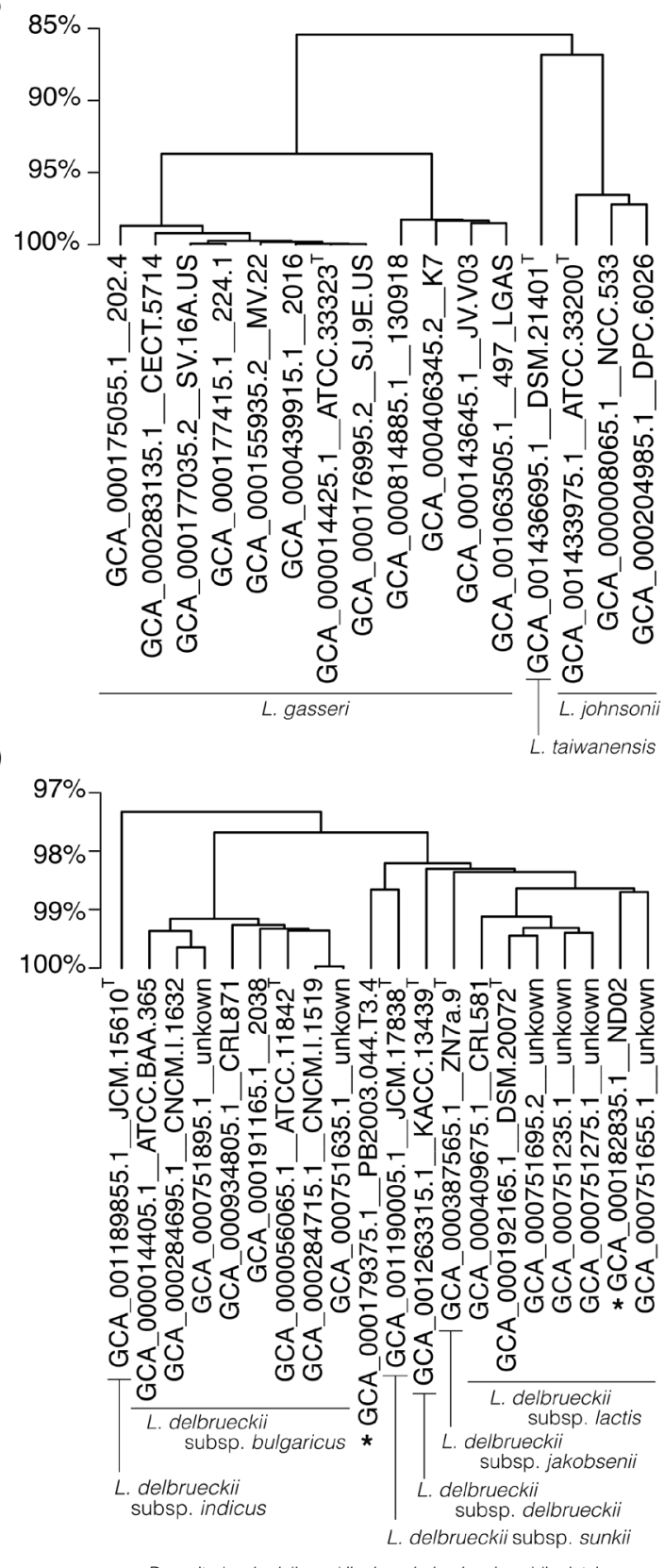

Fig. 3. A) Distribution of ANI values among 191 representative genomes. Circles and open diamonds indicate interspecific and intraspecific ANI values, respectively. Black circles indicate problematic genomes. B-D) Hierarchical clustering results by using $(1-\mathrm{ANI})$ as the genome distance. Each label represents the accession number of NCBI Assembly Database and the strain name. B: Lactobacillus gasseri, C: L. jensenii, D: L. delbrueckii.

de novo from raw reads deposited in SRA. All genomes were annotated by the Prokka (ver. 1.11) pipeline with the customized reference database for LAB. The quality of genomes was assessed by examining the presence of specific gene markers with CheckM, and taxonomic affiliation was verified by ANI. As of January 2016, DAGA covers 168 species and 18 subspecies of the genus Lactobacillus and 11 species of the genus Pediococcus, which correspond to $91 \%$ of the known species for both genera. DAGA utilizes accession numbers from 
the original source as the genome identifiers; data with "GCA" in the genome identifier are from the NCBI Assembly Database, and those with "DRR", "ERR", or "SRR" are from SRA.

Figure 2 shows screenshots of DAGA. Users can query genomes of interest from the search form in the upper part or select a taxonomic name. A keyword search is available too. The genome quality is rated in 5 grades, allowing users to easily select reliable genomes for comparative analysis. The definition of the rating scale and the number of genomes for each grade are shown in Tables 1 and 2. DAGA also provides genome statistics: the number of coding sequences, estimated genome size, and external links to related databases. Annotation results can be downloaded in either GenBank or FASTA format files. DAGA is freely accessible at https://dfast.nig.ac.jp.

\section{Selection of a representative genome for each species}

To verify the taxonomic relationship of each species, we calculated pairwise ANI values among 191 strains representing each species (or subspecies). We gave priority to the type strains in the data selection, and when multiple genomes were available, the one with the highest completeness and the longest average sequence length was chosen. Figure 3A shows the results of ANI calculation (also see our website https://dfast.nig.ac.jp/ download). In most cases, the ANI values between species were below $95 \%$, the threshold to differentiate species. Six strains in Table 3 (black circles in the Fig. 3A) showed anomalously high ANI values, indicating the incongruence of their taxonomic positions, which will be discussed later.

By excluding these six strains, we obtained 185 representative genomes whose interspecific pairwise ANI values were well below $95 \%$. One exception was L. zeae and L. casei, which had an ANI of 94.4\% (see Discussion). After a long period of controversy, L. zeae is now considered to be in the same taxon as $L$. casei [28]. However, the organism name has not been formally rejected in the current nomenclature, and L. zeae was counted with its original name in our database. It should also be noted that the publicly available genome of $L$. amylotrophicus (GCA_001434555.1), which exhibited an ANI of $99.9 \%$ with L. amylophilus, did not serve as the representative genome. Instead, we used data from SRA (ERR387486) as the representative of L. amylotrophicus.

The validity of the 185 representative genomes was also confirmed by comparing their reconstructed $16 \mathrm{~S}$ rRNA gene sequences with those deposited in public databases. When not available, housekeeping genes like pheS or rpoA were used instead. In addition, a phylogenetic tree was constructed using 132 conserved single-copy genes to verify their taxonomic positions, and this tree is available at our website (https://dfast.nig. ac.jp/download/). Selection of representative genomes was implemented as a procedure in our system to serve as a tool for taxonomic studies in which comparison with type strains is critical.

\section{Detection of mislabeled genomes by ANI values}

We next checked the taxonomic affiliation for all genomes in DAGA by conducting ANI calculations against the representative genomes. We adopted species names based on the ANI calculations for 77 mislabeled genomes and inferred names for 55 unidentified genomes that were deposited as Lactobacillus sp. Such genomes with problematic taxonomic positions were marked as Rating 1 (Table 4).

Remarkably, 28 of 32 "L. casei" genomes were in fact L. paracasei, as previously postulated in the literature [29] and indicated by the fact that they shared an ANI of over $98 \%$ with L. paracasei ATCC $25302^{\mathrm{T}}$ and an ANI of less than $85 \%$ with $L$. casei ATCC $393^{\mathrm{T}}$. Among the remaining four " $L$. casei" genomes, two were type strains, one was low quality with $22 \%$ ambiguous bases $(\mathrm{N})$, and the last was the recently published $L$. casei N87 (GCA_001013375.1). The last strain shared an ANI of $96.8 \%$ with L. zeae DSM $20178^{\mathrm{T}}$ and an ANI of $94.3 \%$ with $L$. casei ATCC $393^{\mathrm{T}}$. In the $L$. plantarum group, the members of which are notoriously difficult to identify with $16 \mathrm{~S}$ rRNA sequence similarity, three " $L$. plantarum" genomes were reassigned organism names inferred from ANI results. The strains SNU.Lp177 (GCA_001273585.1), EGD-AQ4 (GCA_000463075.2), and AY01 (GCA_000469115.1) were inferred to be L. plantarum subsp. argentoratensis, L. pentosus, and L. paraplantarum, respectively. All assignments were recorded, i.e., both the original and the corrected names are available in our database.

\section{Genomic diversity of $L A B$ revealed by ANI}

As a demonstrative analysis taking advantage of the wealth of genomic data stored in DAGA, we conducted all-against-all ANI comparison between 704 genomes $(N$ $=704 \times 703 / 2=247,456$ ) to further investigate genomic diversity. Low-quality genomes and genomes with ambiguous taxonomy were excluded. All interspecific ANI values $(N=239,840)$ were less than $95 \%$, while 198 out of the remaining 7,616 intraspecific ANI values were also less than $95 \%$. Such exceptions included the divergence within L. kunkeei, L. gasseri, and L. jensenii. $L$. gasseri and $L$. jensenii were each clearly separated into 
Table 1. Number of genomes deposited in DAGA

\begin{tabular}{ccccccc}
\hline \multirow{2}{*}{ Data source } & \multicolumn{7}{c}{ Quality rating } & \multirow{2}{*}{ Total } \\
\cline { 2 - 6 } & 1 & 2 & 3 & 4 & 5 & 743 \\
\hline DDBJ/ENA/GenBank & 17 & 11 & 59 & 558 & 98 & 678 \\
SRA & 30 & 27 & 4 & 617 & 0 & 1,421 \\
Total & 47 & 38 & 63 & 1,175 & 98 & \\
\hline
\end{tabular}

Table 2. Definition of the quality rating grades

\begin{tabular}{cl}
\hline Quality rating & Definition \\
\hline 5 & High quality complete genomes with completeness $\geq 95 \%$ and contamination $\leq 5 \%$ \\
4 & High quality draft genomes with completeness $\geq 95 \%$ and contamination $\leq 5 \%$ \\
3 & Low quality genomes with completeness $\geq 80 \%$ and contamination $\leq 10 \%$ \\
2 & Disqualified genomes with completeness $<80 \%$ or contamination $>10 \%$ \\
1 & Taxonomically mislabeled or misidentified genomes \\
\hline
\end{tabular}

Table 3. Strains with problematic taxonomic positions

\begin{tabular}{|c|c|c|c|}
\hline Data source* & Organism name & Strain & Description \\
\hline$\overline{\text { GCA_000159175.1 }}$ & Lactobacillus brevis subsp. gravesensis & ATCC $27305^{\#}$ & Shows an ANI value of $97.3 \%$ against $L$. hilgardii. \\
\hline ERR387492 & Lactobacillus fornicalis & $\mathrm{JCM} 12512^{\mathrm{T}}$ & $\begin{array}{l}\text { Shows an ANI value of } 98.7 \% \text { against } L \text {. plantarum } \\
\text { subsp. plantarum. }\end{array}$ \\
\hline GCA_001436985.1 & Lactobacillus homohiochii & DSM $20571^{\mathrm{T}}$ & Shows an ANI value of $99.9 \%$ against $L$. fructivorans. \\
\hline GCA_001434215.1 & Lactobacillus parakefiri & DSM $10551^{\mathrm{T}}$ & $\begin{array}{l}\text { Shows an ANI value of } 99.9 \% \text { against } L \text {. kefiri. Possibly } \\
\text { contaminated with } L \text {. kefiri (contamination value } 98 \% \text { ). }\end{array}$ \\
\hline SRR1561417 & Pediococcus lolii & DSM $19927^{\mathrm{T}}$ & Shows an ANI value of $97.1 \%$ against $P$. acidilactici. \\
\hline GCA_001437265.1 & Pediococcus parvulus & DSM $203321^{\mathrm{T}}$ & $\begin{array}{l}\text { Shows an ANI value of } 92.5 \% \text { against } P \text {. acidilactici. } \\
\text { Possibly contaminated with } P \text {. acidilactici } \\
\text { (contamination value } 98.9 \% \text { ). }\end{array}$ \\
\hline
\end{tabular}

\# Non-type strain.

Table 4. Mislabeled genomes deposited in DDBJ/ENA/GenBank

\begin{tabular}{|c|c|c|c|}
\hline Data source* & Organism name & Strain & Description \\
\hline GCA_000159195.1 & Lactobacillus buchneri & ATCC 11577 & Shows an ANI value of $99.1 \%$ against $L$. hilgardii. \\
\hline GCA_001434555.1 & Lactobacillus amylotrophicus & DSM $20534^{\mathrm{T}}$ & $\begin{array}{l}\text { Shows an ANI value of } 100 \% \text { against } L \text {. amylophilus. } \\
\text { Possibly replaced by the strain of } L \text {. amylophilus. }\end{array}$ \\
\hline GCA_001314245.1 & Lactobacillus gallinarum & HFD4 & Shows an ANI value of $96.7 \%$ against $L$. helveticus. \\
\hline GCA_001273585.1 & Lactobacillus plantarum & SNU.Lp177 & $\begin{array}{l}\text { Shows an ANI value of } 98.9 \% \text { against } L \text {. plantarum } \\
\text { subsp. argentoratensis and an ANI value of } 95.6 \% \\
\text { against subsp. plantarum. }\end{array}$ \\
\hline GCA_001068345.1 & Lactobacillus johnsonii & 987_LJOH & Shows an ANI value of $93.4 \%$ against $L$. gasseri. \\
\hline GCA_001066235.1 & Lactobacillus johnsonii & $770 \_$LJOH & Shows an ANI value of $100 \%$ against $L$. gasseri. \\
\hline GCA_001064985.1 & Lactobacillus helveticus & 459_LHEL & Shows an ANI value of $96.8 \%$ against $L$. gasseri. \\
\hline GCA_001063065.1 & Lactobacillus kefiranofaciens & 249 LKKEF & Shows an ANI value of $100 \%$ against $L$. gasseri. \\
\hline GCA_001063045.1 & Lactobacillus crispatus & 240_LCRI & Shows an ANI value of $100 \%$ against $L$. gasseri. \\
\hline GCA_000469115.1 & Lactobacillus plantarum & AY01 & Shows an ANI value of $99.6 \%$ against $L$. paraplantarum. \\
\hline GCA_000463075.2 & Lactobacillus plantarum & EGD-AQ4 & Shows an ANI value of $92.8 \%$ against $L$. pentosus. \\
\hline GCA_000191545.1 & Lactobacillus acidophilus & $30 \mathrm{SC}$ & Shows an ANI value of $100 \%$ against $L$. amylovorus. \\
\hline GCA_000159195.1 & Lactobacillus buchneri & ATCC 11577 & Shows an ANI value of $99.1 \%$ against $L$. hilgardii. \\
\hline
\end{tabular}

* Those with GCA were derived from NCBI Assembly Database and those with DRR/SRR/ERR were derived from SRA. 
two previously unknown subgroups (Figs. 3B and 3C). The ANI values between the subgroups were $93 \%$ and $88 \%$ for $L$. gasseri and $L$. jensenii, respectively, while the ANI values within the same subgroups were over $98 \%$ in both species. The intraspecific separation was also supported by the multiple alignments of their pheS gene sequences (alignment data not shown). For L. gasseri and $L$. jensenii, the nucleotide identities of pheS genes between the subgroups were $96 \%$ and $93 \%$, while those of rpo $A$ genes were $99 \%$ and $98 \%$, respectively. The intraspecific separation in the two species might deserve subspecies-level differentiation. We must note, however, that our analysis was based on genomic information only. Further analysis including phenotypic characterization is required to establish their valid classifications.

To assess the discriminating power of ANI, ANI values were calculated among six subspecies of $L$. delbrueckii. The ANI values for their type strains were distributed in the range of $97.2-98.4 \%$. In spite of such high values, hierarchical clustering based on the ANI values could separate them (Fig. 3D), and the tree topology was roughly consistent with the ones from multilocus sequence analyses [30,31]. This implies the reliability of ANI in evaluating the genetic subgroups within a species.

\section{DFAST online annotation server}

We developed a web interface for the DFAST annotation pipeline, so that users can manage metadata and submit annotated genomes to DDBJ. Users can annotate their own genomic data by uploading a FASTA formatted file via a submission form and can perform quality and taxonomic assessment using CheckM and the calculation of ANI. A simple annotation editor is also available, allowing users to modify gene product names or gene symbols. Submission files for the DDBJ Mass Submission System are then automatically generated. Results can be downloaded in several formats, including GenBank, Multi-FASTA, and tab-separated formats.

\section{DISCUSSION}

Recent new-generation sequencing technologies are constantly producing more and more genome sequences, making it important to assess their data quality and taxonomic positions. DAGA is a new genome archive that stores quality-controlled and taxonomically confirmed bacterial genomes with consistent annotation. Its quality measure is the genome completeness and contamination values calculated by CheckM, and we were able to use it to successfully identify genomes of incorrect size as compared with typical LAB strains without using any other selection method. In addition, we also identified taxonomically mislabeled genomes in public databases even for type strains (Table 2A). These results will help researchers to select genomes for comparative analysis.

The NCBI Reference Sequence (RefSeq) and the Pathosystems Resource Integration Center (PATRIC) provide consistently annotated genome collections $[32,33]$. They collect genome sequences from DDBJ/ ENA/GenBank and re-annotate them using NCBI Prokaryotic Genome Annotation Pipeline (PGAP) and Rapid Annotation using Subsystem Technology (RAST), respectively. As far as we know, there is no database that collects genomic data from both DDBJ/ENA/GenBank and SRA. Because SRA stores raw sequence data, it is difficult for users without bioinformatics skills to exploit the data. DAGA facilitates the reuse of valuable data available in SRA, such as the only reliable genome for $L$. amylotrophicus, which can only be obtained from SRA (ERR387486).

As of January 2016, DAGA provides 1,421 genomes collected from DDBJ/ENA/GenBank and SRA for two genera in Lactobacillaceae. The genus Sharpea was not included even though it is classified in the family Lactobacillaceae. Sharpea azabuensis, the only member of this genus, was initially described as a species related to Lactobacillus catenaformis, but $L$. catenaformis was later reclassified as Eggerthia catenaformis, and it is no longer a member of Lactobacillaceae [34, 35]. As the number of available genomes is increasing rapidly, we plan to update the database regularly and to expand the scope of the database to other taxonomic groups.

The most widely used methodology for bacterial taxonomic identification is the combination of 16S rRNA gene sequencing and DNA-DNA hybridization (DDH) [36]. According to the minimal standard recommended for describing new species of Lactobacillus, DDH should be conducted if the $16 \mathrm{~S}$ rRNA sequence similarity to the closest known species is beyond 97\% [37]. Recently, however, ANI has been used as a substitute for DDH to describe novel species of Lactobacillus [38-40]. ANI has several advantages. First, it does not require a laboratory assay and is computationally reproducible. Second, it does not require gene calling and is applicable to draft genomes. It is especially valuable in the case of conducting de novo assembly from short reads because bacterial genomes normally encode multiple rRNA operons difficult to reconstruct. Lastly and most importantly, ANI shows prominent discriminatory power to determine genome identity. Even between hard-todistinguish taxonomic groups such as $L$. casei and $L$. plantarum, the ANI values between two different species 
were below $85 \%$, much less than the threshold of $95 \%$. Furthermore, only $0.4 \%$ of the comparisons fell within the "twilight zone" of $85-95 \%$ in our analysis of 191 representative genomes (Fig. 3A). For these reasons, we emphasize the benefit of ANI to validate taxonomic status for genomes deposited in DAGA. As an exception, the ANI value between $L$. casei ATCC $393^{\mathrm{T}}$ and $L$. zeae DSM $20178^{\mathrm{T}}$ was slightly below the species-level threshold (94.4\%) even though the two strains are now considered the same species. In our analysis, ANI values between species were always less than $95 \%$, but the reverse is not always true. As shown by the results for $L$. gasseri and $L$. jensenii, intraspecific ANI values can be lower than $95 \%$ in some species.

In several species, ANI can help determine subspecies of a given strain, as shown in the results for $L$. delbrueckii (Fig. 3D). It seems difficult to establish an ANI cutoff value to distinguish subspecies, however, because intersubspecific ANI values depend on the species (Fig. $3 \mathrm{~A})$. For example, the lowest value exhibited by the subspecies of L. aviarius was $89 \%$, much lower than the species-level threshold. The highest value was reported for L. kefiranofaciens, which showed an ANI value as high as $99.4 \%$. According to the original description, the two subspecies of L. kefiranofaciens shared $100 \%$ 16S rRNA sequence identity and were distinguishable by morphological and biochemical characteristics [41]. On the other hand, the two subspecies of L. plantarum were distinguished mainly based on their genotypic traits because their morphological, physiological, and biochemical characteristics were almost identical, with the only exceptions being in a few carbohydrate fermentation patterns [42]. The ANI value between L. plantarum subsp. plantarum and subsp. argentoratensis was $95.3 \%$. The difference in inter-subspecific ANI values between the two species seems to reflect their original descriptions. For several strains, the allocation of subspecies was found to be inconsistent with the ANI results. L. sakei subsp. sakei $23 \mathrm{~K}$ was more similar to subsp. carnosus than to subsp. sakei, as suggested by Chaillou et al. [43]. The two strains labeled as $L$. paracasei subsp. tolerans (GCA_000409835.1 and GCA_000410335.1) were more similar to subsp. paracasei, although the difference was as small as $0.2 \%$. The genome sizes of subsp. paracasei and subsp. tolerans differ prominently: $3.0 \mathrm{Mbp}$ and 2.4 $\mathrm{Mbp}$, respectively. Judging from the genome sizes, the two strains are more likely to belong to the subspecies paracasei. However, we could not find any other evidence that supports this assumption. The values from all ANI calculations are available from our website: https://dfast. nig.ac.jp/download/.
Our assessment found the six questionable genomes listed in Table 2A, namely, Pediococcus lolii DSM $19927^{\mathrm{T}}$ (GCA_001437115.1), Pediococcus parvulus DSM 203321 $1^{\mathrm{T}} \quad$ (GCA_001437265.1), Lactobacillus brevis subsp. gravesensis ATCC 27305 (GCA 000159175.1), Lactobacillus fornicalis JCM $12512^{\overline{\mathrm{T}}}$ (ERR387492), Lactobacillus homohiochii DSM 20571 ${ }^{\mathrm{T}}$ (GCA_001436985.1), and Lactobacillus parakefiri DSM $10551^{\mathrm{T}}$ (GCA_001434215.1). The $P$. lolii genome was presumably a misclassification of the sequenced strain. A previous study reported that the type strains of P. lolii deposited in DSMZ and JCM were strains of Pediococcus acidilactici [44]. Our analysis showed that not only P. lolii DSM $19927^{\mathrm{T}}$ but also strain NGRI $0510 Q^{\mathrm{T}}$ (GCA_000319265.1), an original type strain of $P$. lolii, shared an ANI of $97 \%$ with P. acidilactici. L. brevis subsp. gravesensis was first described over 60 years ago, but it was not mentioned in the Approved Lists of Bacterial Names published in 1980 [45]. This species is displayed as Lactobacillus sp. and Lactobacillus hilgardii in JCM and the EzGenome database, respectively [12, 46]. The type strains of L. homohiochii and L. fornicalis deposited in culture collections were reported to misrepresent the originally described strains [47] (http:// www.bacterio.net/lactobacillus.html\#fornicalis). Their original strains are no longer available, and designation of a neotype seems appropriate. The genome of $L$. parakefiri DSM 10551 ${ }^{\mathrm{T}}$ (GCA_001434215.1) exhibited an extremely high contamination value (98\%), indicating the mixture of different strains. Indeed, two pheS genes were found in the genome, each matching the deposited pheS gene sequences of $L$. kefiri and L. parakefiri. Our analysis suggests that its large genome size [18] and the similarity to L. kefiri [17] are attributable to the sequence contamination. Likewise, the genome of $P$. parvulus DSM $20332^{\mathrm{T}}$ seems to be contaminated with another strain of $P$. acidilactici.

Our annotation pipeline is freely available as the DFAST web service. In comparison with other annotation tools such as RAST [48] or the Microbial Genome Annotation Pipeline (MiGAP) [49], the advantage of DFAST is the ability to generate ready-to-submit annotation files. RAST can perform detailed functional annotation based on the platform called SEED. However, if users want to submit an annotated genome to INSDC, they need to convert annotation results into an acceptable format. Although MiGAP partly supports the DDBJ-acceptable format, users are still required to prepare metadata and to curate annotated protein names before submission. As our curated reference database follows the protein naming guidelines of the NCBI, minimal manual curation, if any, 
is required before submitting genomes to DDBJ. Another advantage of DFAST is its short running time. It takes about 5 minutes to annotate a typical bacterial genome, while RAST and MiGAP take several hours. In addition, DFAST provides quality and taxonomy assessment tools, which prevent users from submitting low quality or mislabeled genomes to INSDC. We have already used DFAST to annotate 5 genomes of Lactobacillus strains, including two candidates for new species (manuscript in preparation). On average, $90.3 \%$ of protein coding sequences were annotated based on a similarity search against the reference protein database in this study. We were able to submit them to DDBJ without any manual curation. Currently, the reference database constructed in this study is based mainly on protein sequence data obtained from Lactobacillus and Pediococcus, with additional information from 12 representative strains of other genera. Our future tasks include an update and extension of the reference database to other genera, such as Lactococcus and Leuconostoc, and annotation of frameshifted genes or pseudo-genes.

In conclusion, we assessed 1,421 genomes covering 191 (sub)species in the family Lactobacillaceae and developed a curated genome repository referred to as DAGA. This will improve the accessibility and reusability of LAB genome resources. The annotation and submission pipeline DFAST will help researchers to deal with large amounts of emerging sequence data, thereby accelerating studies of LAB that make use of genomic data.

\section{ACKNOWLEDGEMENTS}

We gratefully acknowledge Masanori Tohno and Akihito Endo for helpful discussion and informative suggestions. We also thank Kyungbum Lee and Toshihisa Okido at DDBJ Center for helpful comments. The pyani script was kindly provided by Leighton Pritchard at James Hutton Institute, UK. This work was supported by Collaborative Research Program A (2014-2015) of the National Institute of Genetics (NIG) and the commission for Development of Artificial Gene Synthesis Technology for Creating Innovative Biomaterial of the Ministry of Economy, Trade and Industry, Japan. Computational analysis was performed on the NIG supercomputer at the Research Organization of Information and Systems (ROIS).

\section{REFERENCES}

1. Cochrane G, Karsch-Mizrachi I, Takagi T, International Nucleotide Sequence Database Collaboration. 2016. The International Nucleotide Sequence Database Collaboration. Nucleic Acids Res 44 D1: D48-D50.
[Medline] [CrossRef]

2. Kodama Y, Shumway M, Leinonen R, International Nucleotide Sequence Database Collaboration. 2012. The Sequence Read Archive: explosive growth of sequencing data. Nucleic Acids Res 40: D54-D56. [Medline] [CrossRef]

3. Nilsson RH, Ryberg M, Kristiansson E, Abarenkov K, Larsson KH, Kõljalg U. 2006. Taxonomic reliability of DNA sequences in public sequence databases: a fungal perspective. PLoS One 1: e59. [Medline] [CrossRef]

4. Bull MJ, Marchesi JR, Vandamme P, Plummer S, Mahenthiralingam E. 2012. Minimum taxonomic criteria for bacterial genome sequence depositions and announcements. J Microbiol Methods 89: 18-21. [Medline] [CrossRef]

5. Nakazato T, Ohta T, Bono H. 2013. Experimental design-based functional mining and characterization of high-throughput sequencing data in the sequence read archive. PLoS One 8: e77910. [Medline] [CrossRef]

6. Gurevich A, Saveliev V, Vyahhi N, Tesler G. 2013. QUAST: quality assessment tool for genome assemblies. Bioinformatics 29: 1072-1075. [Medline] [CrossRef]

7. Parks DH, Imelfort M, Skennerton CT, Hugenholtz P, Tyson GW. 2015. CheckM: assessing the quality of microbial genomes recovered from isolates, single cells, and metagenomes. Genome Res 25: 1043-1055. [Medline] [CrossRef]

8. Beaz-Hidalgo R, Hossain MJ, Liles MR, Figueras MJ. 2015. Strategies to avoid wrongly labelled genomes using as example the detected wrong taxonomic affiliation for aeromonas genomes in the GenBank database. PLoS One 10: e0115813. [Medline] [CrossRef]

9. Goris J, Konstantinidis KT, Klappenbach JA, Coenye T, Vandamme P, Tiedje JM. 2007. DNADNA hybridization values and their relationship to whole-genome sequence similarities. Int J Syst Evol Microbiol 57: 81-91. [Medline] [CrossRef]

10. Richter M, Rosselló-Móra R. 2009. Shifting the genomic gold standard for the prokaryotic species definition. Proc Natl Acad Sci USA 106: 19126-19131. [Medline] [CrossRef]

11. Kim M, Oh HS, Park SC, Chun J. 2014. Towards a taxonomic coherence between average nucleotide identity and 16S rRNA gene sequence similarity for species demarcation of prokaryotes. Int J Syst Evol Microbiol 64: 346-351. [Medline] [CrossRef]

12. Kim OS, Cho YJ, Lee K, Yoon SH, Kim M, Na H, Park SC, Jeon YS, Lee JH, Yi H, Won S, Chun J. 2012. Introducing EzTaxon-e: a prokaryotic 16S rRNA gene sequence database with phylotypes that represent uncultured species. Int J Syst Evol Microbiol 62: 716-721. [Medline] [CrossRef]

13. Federhen S, Rosselló-Mora R, Klenk HP, Tindall BJ, Konstantinidis KT, Whitman WB, Brown D, Labeda 
D, Ussery D, Garrity GM, Rita R, Colwell NH, Graf J, Parte A, Yarza P, Goldberg B, Sichtig H, KarschMizrachi I, Clark K, McVeigh R, Pruitt KD, Tatusova T, Falk R, Turner S, Madden T, Kitts P, Kimchi A, Klimke W, Agarwala R, DiCuccio M, Ostell J. 2016. Meeting report: GenBank microbial genomic taxonomy workshop (12-13 May, 2015). Stand Genomic Sci 11: 15. [CrossRef]

14. Sugawara H, Miyazaki S, Gojobori T, Tateno Y. 1999. DNA Data Bank of Japan dealing with large-scale data submission. Nucleic Acids Res 27: 25-28. [Medline] [CrossRef]

15. Zhang ZG, Ye ZQ, Yu L, Shi P. 2011. Phylogenomic reconstruction of lactic acid bacteria: an update. BMC Evol Biol 11: 1. [Medline] [CrossRef]

16. Franz CMAP, Endo A, Abriouel H, Van Reenen CA, Gálvez A, Dicks LMT. 2014. The genus Pediococcus, pp. 359-376. In Holzapfel, WH, Wood, BJB (eds.), Lactic Acid Bacteria: Biodiversity and Taxonomy. John Wiley \& Sons.

17. Zheng J, Ruan L, Sun M, Gänzle M. 2015. A genomic view of lactobacilli and pediococci demonstrates that phylogeny matches ecology and physiology. Appl Environ Microbiol 81: 7233-7243. [Medline] [CrossRef]

18. Sun Z, Harris HMB, McCann A, Guo C, Argimón S, Zhang W, Yang X, Jeffery IB, Cooney JC, Kagawa TF, Liu W, Song Y, Salvetti E, Wrobel A, Rasinkangas P, Parkhill J, Rea MC, O'Sullivan O, Ritari J, Douillard FP, Paul Ross R, Yang R, Briner AE, Felis GE, de Vos WM, Barrangou R, Klaenhammer TR, Caufield PW, Cui Y, Zhang H, O'Toole PW. 2015. Expanding the biotechnology potential of lactobacilli through comparative genomics of 213 strains and associated genera. Nat Commun 6: 8322. [Medline] [CrossRef]

19. Makarova K, Slesarev A, Wolf Y, Sorokin A, Mirkin B, Koonin E, Pavlov A, Pavlova N, Karamychev V, Polouchine N, Shakhova V, Grigoriev I, Lou Y, Rohksar D, Lucas S, Huang K, Goodstein DM, Hawkins T, Plengvidhya V, Welker D, Hughes J, Goh Y, Benson A, Baldwin K, Lee JH, Díaz-Muñiz I, Dosti B, Smeianov V, Wechter W, Barabote R, Lorca G, Altermann E, Barrangou R, Ganesan B, Xie Y, Rawsthorne H, Tamir D, Parker C, Breidt F, Broadbent J, Hutkins R, O’Sullivan D, Steele J, Unlu G, Saier M, Klaenhammer T, Richardson P, Kozyavkin S, Weimer B, Mills D. 2006. Comparative genomics of the lactic acid bacteria. Proc Natl Acad Sci USA 103: 1561115616. [Medline] [CrossRef]

20. Uchiyama I. 2007. MBGD: a platform for microbial comparative genomics based on the automated construction of orthologous groups. Nucleic Acids Res 35: D343-D346. [Medline] [CrossRef]

21. Contreras-Moreira B, Vinuesa P. 2013. GET HOMOLOGUES, a versatile software package for scalable and robust microbial pangenome analysis.
Appl Environ Microbiol 79: 7696-7701. [Medline] [CrossRef]

22. Li L, Stoeckert CJ Jr, Roos DS. 2003. OrthoMCL: identification of ortholog groups for eukaryotic genomes. Genome Res 13: 2178-2189. [Medline] [CrossRef]

23. Marchler-Bauer A, Derbyshire MK, Gonzales NR, Lu S, Chitsaz F, Geer LY, Geer RC, He J, Gwadz M, Hurwitz DI, Lanczycki CJ, Lu F, Marchler GH, Song JS, Thanki N, Wang Z, Yamashita RA, Zhang D, Zheng C, Bryant SH. 2015. CDD: NCBI's conserved domain database. Nucleic Acids Res 43: D222-D226. [Medline] [CrossRef]

24. Seemann T. 2014. Prokka: rapid prokaryotic genome annotation. Bioinformatics 30: 2068-2069. [Medline] [CrossRef]

25. Kitts PA, Church DM, Thibaud-Nissen F, Choi J, Hem V, Sapojnikov V, Smith RG, Tatusova T, Xiang C, Zherikov A, DiCuccio M, Murphy TD, Pruitt KD, Kimchi A. 2016. Assembly: a resource for assembled genomes at NCBI. Nucleic Acids Res 44 D1: D73D80. [Medline] [CrossRef]

26. Kajitani R, Toshimoto K, Noguchi H, Toyoda A, Ogura Y, Okuno M, Yabana M, Harada M, Nagayasu E, Maruyama H, Kohara Y, Fujiyama A, Hayashi T, Itoh T. 2014. Efficient de novo assembly of highly heterozygous genomes from whole-genome shotgun short reads. Genome Res 24: 1384-1395. [Medline] [CrossRef]

27. Altschul SF, Gish W, Miller W, Myers EW, Lipman DJ. 1990. Basic local alignment search tool. J Mol Biol 215: 403-410. [Medline] [CrossRef]

28. Judicial Commission of the International Committee on Systematics of Bacteria. 2008. The type strain of Lactobacillus casei is ATCC 393, ATCC 334 cannot serve as the type because it represents a different taxon, the name Lactobacillus paracasei and its subspecies names are not rejected and the revival of the name 'Lactobacillus zeae' contravenes Rules 51b (1) and (2) of the International Code of Nomenclature of Bacteria. Opinion 82. Int J Syst Evol Microbiol 58: 1764-1765. [Medline] [CrossRef]

29. Smokvina T, Wels M, Polka J, Chervaux C, Brisse S, Boekhorst J, van Hylckama Vlieg JET, Siezen RJ. 2013. Lactobacillus paracasei comparative genomics: towards species pan-genome definition and exploitation of diversity. PLoS One 8: e68731. [Medline] [CrossRef]

30. Tanigawa K, Watanabe K. 2011. Multilocus sequence typing reveals a novel subspeciation of Lactobacillus delbrueckii. Microbiology 157: 727-738. [Medline] [CrossRef]

31. Adimpong DB, Nielsen DS, Sørensen KI, Vogensen FK, Sawadogo-Lingani H, Derkx PMF, Jespersen L. 2013. Lactobacillus delbrueckii subsp. jakobsenii subsp. nov., isolated from dolo wort, an alcoholic 
fermented beverage in Burkina Faso. Int J Syst Evol Microbiol 63: 3720-3726. [Medline] [CrossRef]

32. Tatusova T, Ciufo S, Fedorov B, O'Neill K, Tolstoy I. 2015. RefSeq microbial genomes database: new representation and annotation strategy. Nucleic Acids Res 43: 3872. [Medline] [CrossRef]

33. Wattam AR, Abraham D, Dalay O, Disz TL, Driscoll T, Gabbard JL, Gillespie JJ, Gough R, Hix D, Kenyon R, Machi D, Mao C, Nordberg EK, Olson R, Overbeek R, Pusch GD, Shukla M, Schulman J, Stevens RL, Sullivan DE, Vonstein V, Warren A, Will R, Wilson MJC, Yoo HS, Zhang C, Zhang Y, Sobral BW. 2014. PATRIC, the bacterial bioinformatics database and analysis resource. Nucleic Acids Res 42: D581-D591. [Medline] [CrossRef]

34. Morita H, Shiratori C, Murakami M, Takami H, Toh H, Kato Y, Nakajima F, Takagi M, Akita H, Masaoka T, Hattori M. 2008. Sharpea azabuensis gen. nov., sp. nov., a Gram-positive, strictly anaerobic bacterium isolated from the faeces of thoroughbred horses. Int J Syst Evol Microbiol 58: 2682-2686. [Medline] [CrossRef]

35. Salvetti E, Felis GE, Dellaglio F, Castioni A, Torriani S, Lawson PA. 2011. Reclassification of Lactobacillus catenaformis (Eggerth 1935) Moore and Holdeman 1970 and Lactobacillus vitulinus Sharpe et al. 1973 as Eggerthia catenaformis gen. nov., comb. nov. and Kandleria vitulina gen. nov., comb. nov., respectively. Int J Syst Evol Microbiol 61: 2520-2524. [Medline] [CrossRef]

36. Chun J, Rainey FA. 2014. Integrating genomics into the taxonomy and systematics of the Bacteria and Archaea. Int J Syst Evol Microbiol 64: 316-324. [Medline] [CrossRef]

37. Mattarelli P, Holzapfel W, Franz CMAP, Endo A, Felis GE, Hammes W, Pot B, Dicks L, Dellaglio F. 2014. Recommended minimal standards for description of new taxa of the genera Bifidobacterium, Lactobacillus and related genera. Int J Syst Evol Microbiol 64: 1434-1451. [Medline] [CrossRef]

38. Olofsson TC, Alsterfjord M, Nilson B, Butler E, Vásquez A. 2014. Lactobacillus apinorum sp. nov., Lactobacillus mellifer sp. nov., Lactobacillus mellis sp. nov., Lactobacillus melliventris sp. nov., Lactobacillus kimbladii sp. nov., Lactobacillus helsingborgensis sp. nov. and Lactobacillus kullabergensis sp. nov., isolated from the honey stomach of the honeybee Apis mellifera. Int J Syst Evol Microbiol 64: 3109-3119. [Medline] [CrossRef]

39. Mao Y, Chen M, Horvath P. 2015. Lactobacillus herbarum sp. nov., a species related to Lactobacillus plantarum. Int J Syst Evol Microbiol 65: 4682-4688. [Medline] [CrossRef]

40. Puertas AI, Arahal DR, Ibarburu I, Elizaquível P, Aznar R, Dueñas MT. 2014. Lactobacillus sicerae sp. nov., a lactic acid bacterium isolated from Spanish natural cider. Int J Syst Evol Microbiol 64: 2949-2955. [Medline] [CrossRef]

41. Vancanneyt M, Mengaud J, Cleenwerck I, Vanhonacker K, Hoste B, Dawyndt P, Degivry MC, Ringuet D, Janssens D, Swings J. 2004. Reclassification of Lactobacillus kefirgranum Takizawa et al. 1994 as Lactobacillus kefiranofaciens subsp. kefirgranum subsp. nov. and emended description of $L$. kefiranofaciens Fujisawa et al. 1988. Int J Syst Evol Microbiol 54: 551-556. [Medline] [CrossRef]

42. Bringel F, Castioni A, Olukoya DK, Felis GE, Torriani S, Dellaglio F. 2005. Lactobacillus plantarum subsp. argentoratensis subsp. nov., isolated from vegetable matrices. Int J Syst Evol Microbiol 55: 1629-1634. [Medline] [CrossRef]

43. Chaillou S, Daty M, Baraige F, Dudez AM, Anglade $\mathrm{P}$, Jones $\mathrm{R}$, Alpert $\mathrm{CA}$, Champomier-Vergès $\mathrm{MC}$, Zagorec M. 2009. Intraspecies genomic diversity and natural population structure of the meat-borne lactic acid bacterium Lactobacillus sakei. Appl Environ Microbiol 75: 970-980. [Medline] [CrossRef]

44. Wieme A, Cleenwerck I, Van Landschoot A, Vandamme P. 2012. Pediococcus lolii DSM $19927^{\mathrm{T}}$ and JCM $15055^{\mathrm{T}}$ are strains of Pediococcus acidilactici. Int J Syst Evol Microbiol 62: 3105-3108. [Medline] [CrossRef]

45. Skerman V, McGowan V, Sneath P. 1980. Approved lists of bacterial names. Int J Syst Bacteriol 30: 225420. [CrossRef]

46. Kitahara M. 2008. Quality management of Lactobacillus strains in JCM. Microbiol Cult Collect 24: 143-145.

47. Goto N, Joyeux A, Lonvaud-Funel A. 1994. Taxonomic problem of the type strain of Lactobacillus homohiochii. J Brew Soc Jpn 89: 643-646. [CrossRef]

48. Overbeek R, Olson R, Pusch GD, Olsen GJ, Davis JJ, Disz T, Edwards RA, Gerdes S, Parrello B, Shukla M, Vonstein V, Wattam AR, Xia F, Stevens R. 2014. The SEED and the Rapid Annotation of microbial genomes using Subsystems Technology (RAST). Nucleic Acids Res 42: D206-D214. [Medline] [CrossRef]

49. Sugawara H, Ohyama A, Mori H, Kurokawa K. 2009. Microbial genome annotation pipeline (MiGAP) for diverse users. In Proceedings of the 20th International Conference on Genome Informatics, Yokohama, pp. S-001-1-2. 